\title{
In vitro assessment of metabolic profile of Enterococcus strains of human origin
}

\author{
Ashlesha Bhagwat and Uday S. Annapure*
}

\begin{abstract}
Background: In the present study, previously isolated, safe, and avirulent enterococci strains were exploited for their metabolic profile (Bhagwat et al., Asian J Pharm Clin Res 12: 2019).

Results: Thirteen enterococci strains of human origin produced important enzymes like amylase $\left(0.5-0.7 \mathrm{mg} \mathrm{ml}^{-1}\right)$, protease $\left(192-264 \mathrm{mg} \mathrm{ml}^{-1}\right)$, lipase $\left(8-10 \mathrm{mg} \mathrm{ml}^{-1}\right)$, bile salt hydrolase, conjugated linoleic acid (CLA), and lactic acid (highest $12 \mathrm{mg} \mathrm{ml}^{-1}$ ), thus implicating potential attributes of starter cultures in food and dairy industry. Biogenic amines like arginine and tryptamine were produced after 4 days above $25^{\circ} \mathrm{C}$. Castor oil (highest yield $60 \mu \mathrm{mll}^{-1}$ ) and sunflower oil (highest yield $48 \mathrm{\mu g} \mathrm{ml}^{-1}$ ) both proved to be excellent sources of CLA production. Reduction assays using FRAP, ABTS (above 83\%), and DPPH (30-50\%) revealed excellent radical scavenging properties of cellfree supernatants of Enterococcus strains.
\end{abstract}

Conclusion: The results implicate the future potential of application enterococci for therapeutic purpose as well as the food industry.

Keywords: Enterococcus, Enzymes, BA, BSH, CLA, Antioxidant

\section{Background}

The gut microbiota is very diverse and has varied metabolic activities. These activities are useful as well as harmful to the host. The potential of the probiotic organism depends on the beneficial secondary metabolites produced by them. They are known to produce various enzymes like $\beta$-galactosidase, proteases, lipases, and bacteriocins that possess antimicrobial activity. $\beta$ galactosidase helps in digesting the lactose which in turn helps in healing the lactose-intolerant people. Proteases and lipases help in the formation of biofilms, in turn, improving the process of pathogen exclusion. These enzymes also help in reducing hypertension [2, 3]. Hence, the screening of probiotics considers metabolite production in their selection criteria $[4,5]$.

Probiotic strains are also widely used as starter cultures in the food and dairy industry for, e.g., cheese ripening, wine, beer making. Several lactic acid bacteria are known to produce biogenic amines (BA). These amines are produced from the decarboxylase activity of the organism. They are organic, basic, and nitrogenous toxic

\footnotetext{
* Correspondence: udayannapure@gmail.com

Department of Food Engineering and Technology, Institute of Chemical Technology, Mumbai 400019, India
}

substances that produce toxic amines from proteinaceous substances. Some of the important amines are putrescine, cadaverine, histamine, and agmatine and produced from ornithine, lysine, histidine, arginine, and tyrosine, respectively. The presence of BA in foods in higher concentrations indicates undesirable microbial activity in food. BA are present in the human body in small amounts and involved in biological processes like an immune response, synaptic transmission, and cell growth. But, the consumption of BA in large amounts can be harmful. Hence, it is necessary to prevent its formation in food products. The lactic acid bacteria produce BA under specific temperature and conditions; hence, a strain should only be passed if the strain does not produce the amine at the required temperature [6, 7].

Conjugated linoleic acids (cis-9, trans-11-CLA and trans-10, cis-12-CLA) are isomers naturally found in milk, tissue-containing fat. It is a biogenic isomer essentially a mixture of geometric and positional isomers of linoleic acid (C18:2). Recent studies have reported their multiple properties like anticarcinogenic, anti-obesity, and immune-modulatory activities [8-11]. Owing to their numerous health benefits, there has been an increase in research related to producing these isomers 
with the help of human-derived starter cultures in the food industry. Different strains of lactobacilli, bifidobacteria, and Propionibacterium have revealed their bioactive fatty acidproducing properties in milk or synthetic media [12].

Today, enterococci have created interest in the field of probiotics. The use of enterococci in foods is now a cause of concern due to the presence of virulent enterococci in nosocomial infections. According to FAO and WHO, the possession of virulent genes does not generalize the organism to be pathogenic. Enterococci like E. faecium M74 and E. faecium SF68 are in commercial use in animal feed and have proved their probiotic importance $[13,14]$. Currently, there is a need to assess the specific bioactivities of microorganisms in intestinal microbiology. Hence, the present investigation attempted at screening of enterococci strains for their beneficial attributes. The screening will also help to detect industrially important enzymes of the food industry.

\section{Methods}

\section{Bacterial strains and growth conditions}

Healthy human vagina and gastrointestinal tract are important sources of gut-stabilizing beneficial bacteria. Maternal microbiota is the foundation of gut development of the neonate. Thirteen Enterococcus strains used in the study were isolated from the healthy human vagina and fresh meconium of the neonates and identified by $16 \mathrm{~S}$ rRNA sequencing and submitted to NCBI GenBank under accession numbers KX830968-KX830982. The study was approved by the Ethics Committee of Lokmanya Tilak General Municipal Corporation Hospital, Mumbai, India. The nonvirulent strains were previously characterized for in vitro probiotic aspects [1]. The study involved strains indigenous to human source as human origin strains are preferred for probiotic use according to the criterion laid down by Food and Drug Administration (FDA). The strains were grown in MRS broth at $37^{\circ} \mathrm{C}$ aerobically for $18 \mathrm{~h}$, preserved in $40 \%$ glycerol stocks at $-20^{\circ} \mathrm{C}$ and sub-cultured periodically. All the strains were sub-cultured in MRS broth before use for each assay. The commercially well-known probiotic strain Lactobacillus casei Shirota (L. casei YIT 9029) was used as a reference strain for comparison for all the assays.

\section{Starch, Tween $\mathbf{8 0}$, and cellulose hydrolysis}

The amylolytic and cellulolytic activity was checked by spot inoculating the strain on MRS agar supplemented with $1 \%$ starch and $1 \%$ carboxy methyl cellulose following incubation at $30^{\circ} \mathrm{C}$, for 4-5 days. Lugol's solution was added and spread all over the plate and the halo created due hydrolysis of starch and cellulose was measured [15]. The lipolytic activity was checked by streaking culture on MRS medium supplemented with 1\% Tween 80 following incubation at $30^{\circ} \mathrm{C}$ for $4-5$ days. Precipitation with a halo around the colonies indicated lipolytic activity [16].

\section{Gelatinase, urease, and oxidase activity}

The culture strain was stab inoculated into gelatin medium (peptone $5 \mathrm{gl}^{-1}$, beef extract $3 \mathrm{gl}^{-1}$, gelatin $120 \mathrm{gl}^{-1}$ ) and incubated at $37^{\circ} \mathrm{C}$ for 5 days. The tubes were then kept at $10{ }^{\circ} \mathrm{C}$ for $15-20 \mathrm{~min}$ and observed for gelatin liquefaction by tilting the tube [17]. The urease activity was checked by inoculating strains in Christenson's urea agar plates with phenol red as the indicator. A pink zone around the colony indicated urease activity [18]. For oxidase activity, a well-isolated colony of the test culture was spread on the oxidase disc. A blue color change within $15-30 \mathrm{~s}$ was reported as a positive reaction for the presence of oxidase enzyme $[19,20]$.

\section{Hippurate and esculin hydrolysis}

A hippurate impregnated disc was added to $\mathrm{BHI}$ broth with the test culture and incubated at $37^{\circ} \mathrm{C}$ for $24 \mathrm{~h}$. Two milliliters of the supernatant obtained by centrifugation was mixed with $2 \mathrm{ml}$ of ferric chloride reagent. Precipitation persisting for more or equal to $10 \mathrm{~min}$ indicated the presence of hippurate enzyme [21]. For esculin hydrolysis, 18-h-old test culture was swabbed and esculin discs were embedded on bile containing agar, incubated at $37^{\circ} \mathrm{C}$ for $72 \mathrm{~h}$. Black precipitation around the disc indicated esculinase activity [22].

\section{Deconjugation of bile salts}

For this test, MRS agar supplemented with $0.5 \%$ taurodeoxycholic acid and $0.37 \%$ calcium chloride was poured into petri plates and placed in anaerobic jars for at least $72 \mathrm{~h}$ before use. Wells were cut and inoculated with $10 \mu \mathrm{l}$ 18-h-old culture. The plates were then incubated anaerobically at $37^{\circ} \mathrm{C}$ for $72 \mathrm{~h}$. Highly active strains precipitated bile in $48 \mathrm{~h}$. Precipitation halo around the colonies was measured for bile deconjugation activity. Lactobacillus casei Shirota and E. coli ATCC 8739 were used as positive control and negative control, respectively [23].

\section{Production of biogenic amines}

An improved method was applied to check the decarboxylase activity of the strains. The decarboxylase activity was checked by inoculating various amino acids in the Moeller's decarboxylase medium (Sigma Chemicals, USA). The amino acid discs of ornithine, lysine, tyrosine, proline, histidine, arginine, and serine were used for detecting biogenic amine activity. The disc was inoculated into the broth and overlaid with paraffin oil and incubated at $10^{\circ} \mathrm{C}, 20^{\circ} \mathrm{C}, 25^{\circ} \mathrm{C}$, and $37^{\circ} \mathrm{C}$ for 10 days, respectively. The color change from yellow to purple was scored as positive for the amine production [24].

\section{Protease, amylase, and lipase activity}

The protease activity was evaluated by using casein as the substrate as described by Nguyen et al. One unit of 
protease activity was determined by the amount of enzyme required to release TCA-soluble fragment giving blue color equivalent to $1 \mu \mathrm{g}$ of tyrosine [25].

The alpha-amylase activity of enterococci was determined by DNSA (dinitro salicylic acid) method. The supernatant of cells grown in BHI medium was obtained by centrifugation $\left(8000 \mathrm{rpm}, 15 \mathrm{~min}, 4^{\circ} \mathrm{C}\right)$. The crude supernatant was then used for determining the amylase activity. One unit of an enzyme is defined as the amount of enzyme that allows the hydrolysis of $10 \mathrm{mg}$ of starch for 15 min under given conditions [26].

For lipase assay, test culture was grown in MRS broth supplemented with $1 \%$ tributyrin for $48 \mathrm{~h}$ at $37^{\circ} \mathrm{C}$. The culture was centrifuged $\left(8000 \mathrm{rpm}, 10 \mathrm{~min}, 4^{\circ} \mathrm{C}\right)$, resuspended in $0.05 \mathrm{M}$ of PBS (pH 6.5), and sonicated to obtain cell-free supernatant. The substrate used for analysis consisted of $5 \%$ polyvinyl alcohol mixed with 20 $\mathrm{ml}$ of olive oil and $80 \mathrm{ml}$ of double distilled water. The mixture was stirred overnight and sterilized prior to use. Then, $2.5 \mathrm{ml}$ of the substrate with $2 \mathrm{ml}$ of buffer and 0.5 $\mathrm{ml}$ of supernatant was incubated at $37^{\circ} \mathrm{C}$ for $20 \mathrm{~min}$. The reaction was terminated by adding $10 \mathrm{ml}$ acetone. Distilled water was used as a control in the assay. A unit of lipase enzyme can be defined as the amount of $0.05 \mathrm{M}$ $\mathrm{NaOH}$ required to neutralize the released after hydrolysis of fatty acids per $1 \mathrm{ml}$ of enzyme per minute [27].

\section{$\beta$-galactosidase activity}

The cell pellet was obtained by centrifuging 18-h-old culture at $6000 \mathrm{rpm}, 4^{\circ} \mathrm{C}$ for $10 \mathrm{~min}$. The pellet was washed twice, centrifuged again, and resuspended in PBS $(0.01 \mathrm{M}$, $\mathrm{pH}$ 7). The cells were sonicated or homogenized using mortar and pestle at $4{ }^{\circ} \mathrm{C}$. The resulting supernatant was then filtered to obtain a cell-free supernatant. A single ONPG disc was added to $1 \mathrm{ml}$ of the supernatant and incubated for $30 \mathrm{~min}$ at $37^{\circ} \mathrm{C}$. The reaction was stopped by adding 2 $\mathrm{ml} \mathrm{Na}_{2} \mathrm{CO}_{3}(0.6 \mathrm{M})$. $\beta$-galactosidase cleaves ONPG to release ortho-nitrophenol. Its content was measured by noting the absorbance at $420 \mathrm{~nm}$ for $0,30,60$, and $90 \mathrm{~min}$ interval time [28].

\section{Conjugated linoleic acid production}

An improved rapid method was employed for CLA producing enterococci. Briefly, test cultures were inoculated in MRS broth supplemented with free linoleic acid $(0.5 \mathrm{mg}$ $\mathrm{ml}^{-1}$ ) and castor oil (1\%) and sunflower oil (1\%) followed by incubation at $37^{\circ} \mathrm{C}$ for $48 \mathrm{~h}$. One percent Tween 80 was added to each tube for production of lipase and free availability of linoleic acid from the substrates. The culture broth $(1 \mathrm{ml})$ was centrifuged at 20,800 $\mathrm{g}$ for $1 \mathrm{~min}$ and the pellet was discarded. The supernatant was mixed with 2 $\mathrm{ml}$ of isopropanol, vortexed and rested for $3 \mathrm{~min}$, following acid extraction by adding $1.5 \mathrm{ml}$ hexane. The CLA content was determined spectrophotometrically at $233 \mathrm{~nm}$ by dispensing $230 \mu \mathrm{l}$ of fat-soluble hexane layer in microtiter plate [29].

\section{Lactic acid production}

Briefly, $25 \mathrm{ml}$ of test broth culture was titrated with 0.1 $\mathrm{N} \mathrm{NaOH}$ using $1 \mathrm{ml}$ phenolphthalein as the indicator until the color change to pink. The lactic acid content was calculated according to AOAC standards $(1 \mathrm{ml}$ $\mathrm{NaOH}=90.08 \mathrm{mg}$ of lactic acid) $[15,30]$.

\section{Determination of reduction activity by ferric reducing antioxidant power (FRAP)}

The culture was centrifuged at $8000 \mathrm{rpm}$ for $10 \mathrm{~min}$ at $4{ }^{\circ} \mathrm{C}$. The cell-free supernatant was obtained and filtered through $0.22 \mu$ Merck-Millipore filter and used for antioxidant assays. Briefly, $0.5 \mathrm{ml}$ of cell-free supernatant was mixed with $0.5 \mathrm{ml}$ of $0.2 \mathrm{M}$ sodium phosphate buffer (pH 6.6) and $0.5 \mathrm{ml}$ of $1 \%$ potassium ferricyanide $\left(\mathrm{w} \mathrm{v}^{-1}\right)$. The above mixture was incubated at $50^{\circ} \mathrm{C}$ for $20 \mathrm{~min}$. The solution was then cooled rapidly and $0.5 \mathrm{ml}$ of $10 \%$ TCA $\left(\mathrm{w} \mathrm{v}^{-1}\right)$ was added. The mixture was then centrifuged at $3000 \mathrm{rpm}$ for $5 \mathrm{~min}$. One milliliter of the upper layer of the mixture was mixed with $1 \mathrm{ml}$ of $0.1 \% \mathrm{FeCl}_{3}\left(\mathrm{w} \mathrm{v}^{-1}\right)$. The absorbance was noted after $10 \mathrm{~min}$ at $700 \mathrm{~nm}$. Distilled water was used as blank for the experiment. Trolox was used as a standard for reducing power activity. A higher absorbance indicated higher reducing power. Trolox $\left(0.5 \mathrm{mg} \mathrm{ml}^{-1}\right)$ was used as the standard [31].

\section{DPPH free radical scavenging assay}

The DPPH (2,2-diphenyl-1-picrylhydrazyl) assay is based on the scavenging of DPPH radicals leading to a decrease in the absorbance at $517 \mathrm{~nm}$. The cell-free supernatant was 1:10 diluted with distilled water $(\mathrm{D} / \mathrm{W})$ before use. Around $40 \mu \mathrm{l}$ of cell-free supernatant was mixed with $140 \mu \mathrm{l}$ of methanol and $40 \mu \mathrm{l} \mathrm{DPPH}(0.15 \mathrm{mM})$. The absorbance was noted at $517 \mathrm{~nm}$. Trolox $\left(0.5 \mathrm{mg} \mathrm{ml}^{-1}\right)$ was used as a standard in the assay [32].

The percentage inhibition was calculated by the following formula:

$$
\text { DPPH scavenging activity }=\frac{(\text { Absorbance }) t=0-(\text { Absorbance }) t=15}{(\text { Absorbance }) t=0} \times 100,
$$

where $t_{0}=$ absorbance at $0 \mathrm{~min}$ and $t_{15}=$ absorbance after $15 \mathrm{~min}$.

\section{ABTS free radical scavenging assay}

The ABTS radical scavenging activity was measured 734 nm using Hitachi Spectrophotometer. The reagent was prepared by mixing $7 \mathrm{mM}$ ABTS in water with $2.45 \mathrm{mM}$ potassium persulfate to produce the radical. This mixture was allowed to stand for $16 \mathrm{~h}$ in the dark before use. The reagent was freshly prepared and used within 3 
days. The ABTS stock solution was diluted with methanol and set to an absorbance of $0.7 \pm 0.020$ at $734 \mathrm{~nm}$. The supernatants obtained after centrifugation were diluted (1:10) with methanol to obtain inhibition in the range of $20-95 \%$. Trolox $\left(0.5 \mathrm{mg} \mathrm{m}^{-1}\right)$ was used as standard for the assay. Forty microliters of test supernatant was mixed with $260 \mu \mathrm{l}$ of ABTS solution and the absorbance was noted after $6 \mathrm{~min}$ at $734 \mathrm{~nm}$. The lowest absorbance indicates the highest antioxidant activity. The percentage inhibition was calculated by the following formula:

$$
\text { Percent inhibition }=1-\frac{A s}{A c} \times 100 \text {; }
$$

where $A_{c}=$ absorbance of control and $A_{s}=$ absorbance of sample [33, 34].

\section{Statistical analysis}

All the assays were performed in triplicate. The Statistical Package for Social Sciences (SPSS) software for Windows version (16.0) was used to compare the strains using analysis of variance (ANOVA). Post hoc tests like Duncan were approached to show the significance at $p<0.05$.

\section{Results}

\section{Qualitative detection of enzymes}

All the enterococcal strains produced a zone of precipitation after adding iodine. The starch hydrolysis activity was displayed by all the enterococcal strains revealing amylase production (Table 1). Also, all the strains hydrolyzed Tween 80 by producing precipitation zones around the colonies indicating lipase activity. Gelatinase and cellulase activity was absent in the tested Enterococcus strains. Protease activity was displayed by all the strains by hydrolyzing casein. Strains E. phoeniculicola S20A, E. dispar S16B, E. canintestini AB1, and E. dispar S27A were bile-esculin positive. All the strains were hippurate non-hydrolyzing, urease and oxidase negative. The results can be related with Enterococcus spp. identification key [35].

\section{Deconjugation of bile salts}

The bile salt precipitation zone was measured and categorized accordingly. The precipitation zone is formed by the bile salt hydrolase (BSH) enzyme by breaking down the bile salt. All the strains displayed high BSH activity (Table 2).

\section{Production of biogenic amines}

The strains were screened for the production of biogenic amines. All the enterococcal strains produced tyramine and agmatine after 3-4 days of incubation at $25^{\circ} \mathrm{C}$ and $37^{\circ} \mathrm{C}$. Biogenic amine formation was not observed at 4 , 12 , and $20^{\circ} \mathrm{C}$ (Table 3). Putrescine, tyramine, and cadaverine production was observed in the control strain $L$. casei Shirota. The results reveal that the amines formed only above $25^{\circ} \mathrm{C}$. Similar results were observed in the case to identify an enterococcal population in milk. The authors reported the presence of these genes tyr and $\arg$ in the enterococcal strains. They also found that these enzymes were specifically produced in protein-rich foods and also was particularly temperature dependent [6].

\section{Protease, amylase, and lipase activity}

The proteolytic activity was displayed by all the strains in range of $192-264 \mathrm{mg} \mathrm{ml}^{-1}$. Strains E. phoeniculicola SB3 and E. canintestini S20A produced the highest

Table 1 Qualitative study of hydrolysis of various substrates by Enterococcus strains

\begin{tabular}{|c|c|c|c|c|c|c|c|c|c|c|}
\hline Strains & Starch & Tween 80 & Cellulose & Gelatin & Casein & Esculin & Urea & Hippurate & Oxidase & $\beta$-galactosidase \\
\hline$\overline{\mathrm{AB2}}$ & + & + & - & - & + & - & - & - & - & - \\
\hline SB2 & + & + & - & - & + & - & - & - & - & - \\
\hline S20A & + & + & - & - & + & + & - & - & - & - \\
\hline S14B & + & + & - & - & + & - & - & - & - & - \\
\hline$A B 1$ & + & + & - & - & + & + & - & - & - & - \\
\hline S16B & + & + & - & - & + & + & - & - & - & - \\
\hline $\mathrm{S} 22 \mathrm{C}$ & + & + & - & - & + & - & - & - & - & - \\
\hline S18A & + & + & - & - & + & - & - & - & - & - \\
\hline SB3 & + & + & - & - & + & - & - & - & - & - \\
\hline S27A & + & + & - & - & + & + & - & - & - & - \\
\hline S14B & + & + & - & - & + & - & - & - & - & - \\
\hline S26B & + & + & - & - & + & - & - & - & - & - \\
\hline$S 20 B$ & + & + & - & - & + & - & - & - & - & - \\
\hline LCS & + & + & - & - & + & - & - & - & - & - \\
\hline
\end{tabular}

+ , hydrolysis; -, no hydrolysis 
Table 2 Bile salt hydrolase activity

\begin{tabular}{lll}
\hline Strains & Precipitation zone $(\mathrm{mm})$ & BSH activity \\
\hline SB3 & $16.7 \pm 0.42$ & $\mathrm{H}$ \\
AB1 & $18.65 \pm 0.49$ & $\mathrm{H}$ \\
SB2 & $18.3 \pm 0.14$ & $\mathrm{H}$ \\
S18A & $19.85 \pm 0.21$ & $\mathrm{H}$ \\
S26A & $15.2 \pm 0.28$ & $\mathrm{H}$ \\
S20B & $13.85 \pm 0.21$ & $\mathrm{H}$ \\
S22C & $19.3 \pm 0.28$ & $\mathrm{H}$ \\
S14B & $17.9 \pm 0.24$ & $\mathrm{H}$ \\
S20A & $17.2 \pm 0.19$ & $\mathrm{H}$ \\
S14B & $18.65 \pm 0.36$ & $\mathrm{H}$ \\
S27A & $17.8 \pm 0.31$ & $\mathrm{H}$ \\
S16B & $19.3 \pm 0.26$ & $\mathrm{H}$ \\
SB2 & $19.4 \pm 0.19$ & $\mathrm{H}$ \\
LCS & $16.6 \pm 0.37$ & $\mathrm{H}$ \\
E.coli & $2.30 \pm 0.21$ & $\mathrm{~L}$ \\
\hline
\end{tabular}

Values reported are mean \pm S.D. of three independent experiments $\mathrm{H}$; high (10-20 mm), M; medium (5-10 mm), L; very low/negligible activity $(0-5 \mathrm{~mm})$ $H$ high $(10-20 \mathrm{~mm}), M$ medium $(5-10 \mathrm{~mm}), L$ very low/negligible activity $(0-5 \mathrm{~mm})$

amount of protease enzyme $\left(264 \mathrm{mg} \mathrm{ml}^{-1}\right)$ as compared to $L$. casei Shirota $\left(200 \mathrm{mg} \mathrm{ml}^{-1}\right)$. Amylase enzyme was also produced by all the strains (Table 4). The amylase enzyme was secreted by all the strains in the range of $0.5-0.7 \mathrm{mg} \mathrm{ml}^{-1}$. Strain E. canintestini $\mathrm{AB} 1$ exhibited the highest amylolytic activity $\left(0.7 \mathrm{mg} \mathrm{ml}^{-1}\right)$ followed by $E$. canintestini AB2. The enterococcal strains were screened for their lipase activity by using tributyrin as lipase

Table 3 Detection of biogenic amines

\begin{tabular}{|c|c|c|c|c|c|c|}
\hline \multirow[t]{2}{*}{ Strain } & \multicolumn{6}{|c|}{ Amino acids } \\
\hline & Lysine & Ornithine & Arginine & Histidine & Serine & Tyrosine \\
\hline S20A & $-{ }^{b}$ & - & $+^{a}$ & - & - & + \\
\hline S27A & - & - & + & - & - & + \\
\hline SB3 & - & - & + & - & - & + \\
\hline SB2 & - & - & + & - & - & + \\
\hline$S 20 B$ & - & - & + & - & - & + \\
\hline S26B & - & - & + & - & - & + \\
\hline S22C & - & - & + & - & - & + \\
\hline S16B & - & - & + & - & - & + \\
\hline S18A & - & - & + & - & - & + \\
\hline AB2 & - & - & + & - & - & + \\
\hline AB1 & - & - & + & - & - & + \\
\hline S14B & - & - & + & - & - & + \\
\hline S26A & - & - & + & - & - & + \\
\hline LCS & + & + & - & - & - & + \\
\hline
\end{tabular}

aiogenic amine production detected above $25^{\circ} \mathrm{C}$ after 4 days of incubation ${ }^{\mathrm{b}}$ Not detected
Table 4 Amylase and protease enzyme production by enterococcal strains

\begin{tabular}{lll}
\hline Strains & Amylase $\left(\mathrm{mg} \mathrm{ml}^{-1}\right)$ & Protease $\left(\mathrm{mg} \mathrm{ml}^{-1}\right)$ \\
\hline S26A & $0.6 \pm 0.2^{\mathrm{b}}$ & $212 \pm 3.8^{\mathrm{d}}$ \\
S27A & $0.54 \pm 0.08^{\text {de }}$ & $200 \pm 3.5^{\mathrm{f}}$ \\
SB2 & $0.56 \pm 0.07^{\mathrm{b}}$ & $208 \pm 2.3^{\mathrm{de}}$ \\
AB2 & $0.63 \pm 0.05^{\mathrm{b}}$ & $240 \pm 2.6^{\mathrm{c}}$ \\
SB3 & $0.6 \pm 0.10^{\mathrm{b}}$ & $264 \pm 1.9^{\mathrm{a}}$ \\
S20A & $0.56 \pm 0.2^{\text {cd }}$ & $264 \pm 2.5^{\mathrm{a}}$ \\
S26B & $0.54 \pm 0.09^{\text {de }}$ & $248 \pm 1.9^{\mathrm{b}}$ \\
S22C & $0.53 \pm 0.06^{\mathrm{e}}$ & $208 \pm 2.4^{\mathrm{e}}$ \\
S16B & $0.58 \pm 0.12^{\text {bc }}$ & $240 \pm 3.5^{\mathrm{c}}$ \\
S18A & $0.58 \pm 0.07^{\mathrm{bc}}$ & $192 \pm 3^{\mathrm{g}}$ \\
S20B & $0.54 \pm 0.2^{\text {de }}$ & $212 \pm 3.4^{\mathrm{d}}$ \\
AB1 & $0.7 \pm 0.08^{\mathrm{a}}$ & $212 \pm 2.5^{\mathrm{d}}$ \\
S14B & $0.56 \pm 0.06^{\text {cd }}$ & $204 \pm 3.6^{\text {ef }}$ \\
LCS & $0.54 \pm 0.15^{\text {de }}$ & $200 \pm 2.8^{\mathrm{f}}$ \\
\hline
\end{tabular}

Values reported are mean \pm SD of triplicate experiments. Values within each row and column with different letter assigned are significantly different $p<0.05$

enzyme inducer. All the tested strains exhibited excellent lipase production in the range of $8-9 \mathrm{U} \mathrm{ml}^{-1}$. Strain $E$. dispar S26A showed the highest amount of enzyme activity of $9.4 \mathrm{U} \mathrm{ml}^{-1}$ greater than the L. casei Shirota strain. Figure 1 shows the lipase production of strains.

\section{$\beta$-galactosidase activity}

The $\beta$-galactosidase activity was absent in the enterococci strains (Table 1). Not all enterococcal strains are capable of producing this important enzyme. The absence of these enzymes does not mean that they are not fit for probiotic use. However, the presence of enzymes like amylase, protease, and lipase may prove their usefulness in the GIT.

\section{Conjugated linoleic acid production}

The production of conjugated linoleic acid from naturally rich substrates like castor oil and sunflower oil is graphically presented in Fig. 2. E. dispar S20B shows the highest conversion activity $\left(60 \mu \mathrm{g} \mathrm{ml}^{-1}\right)$ from castor oil. Strains E. dispar S20B, E. rivorum S22C, E. dispar S16B, E. villorum $\mathrm{SB} 2, E$. canintestini $\mathrm{S} 26 \mathrm{~B}$, and standard $L$. casei Shirota produced $\geq 40 \mu \mathrm{g} \mathrm{ml}^{-1}$ from castor oil and sunflower oil. Kolaglu et al. reported about CLA production of about $33.5 \pm 1.74 \mathrm{mg} 100 \mathrm{~g}^{-1}$ by L. lactis subspp. Lactis and L. reuteri.

\section{Lactic acid production}

The lactic acid production profile is elucidated graphically in Fig. 3. E. rivorum S22C shows the highest yield of lactic acid. The rest of the strains varied production values $\left(7-10 \mathrm{mg} \mathrm{ml}^{-1}\right)$. 


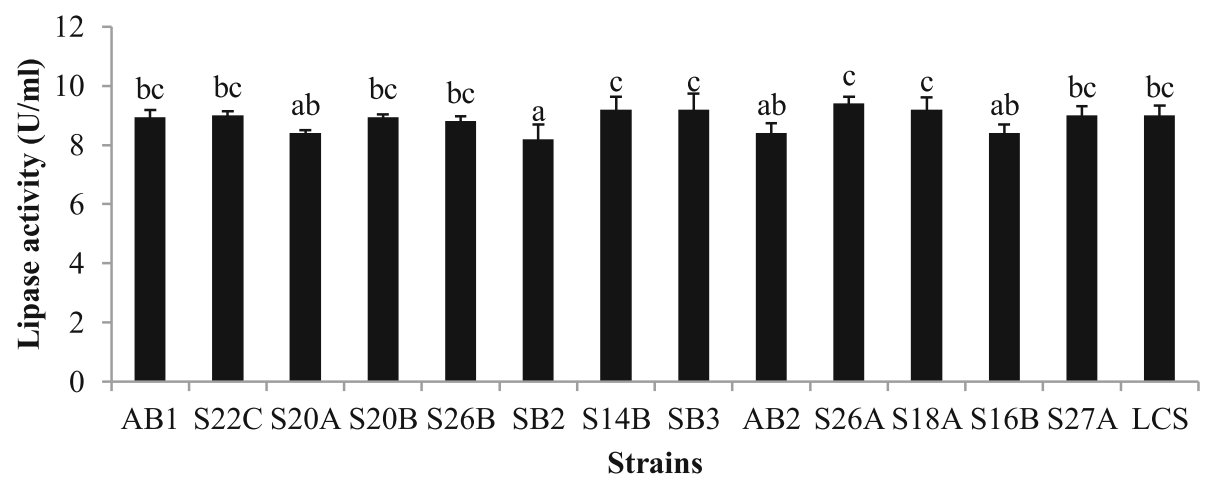

Fig. 1 Lipase activity of the Enterococcus strains. The strains are AB1; E. canintestini AB1, S22C; E. rivorum S22C, S20A; E. phoeniculicola S20A, S20B; E. dispar S20B, S26B; E. canintestini S26B, SB2; E. villorum SB2, S14B; E. rivorum S14B, SB3; E. canintestini SB3, AB2; E. canintestini AB2, S26A; E. dispar S26A, S18A; E. canintestini S18A, S16B; E. dispar S16B, S27A; E. dispar S27A, LCS; and L. casei Shirota, respectively. Columns with different letters are significantly different $p<0.05$

\section{Antioxidant activity of Enterococcus strains}

It is difficult to assess antioxidant activity by a single method owing to the irregularity of radical scavenging systems. Hence, it is suggested that one should determine the activity by using two or more methods. In this study, the radical scavenging property was assessed by DPPH, FRAP, and ABTS assays. Trolox, a known synthetic antioxidant, was used for comparing the activity of the strains in this study.

The reducing power of extracellular extract was determined by using potassium ferricyanide. The reducers present in the test solution reduce the ferricyanide complex into the ferrous form. This reduction causes a change in color from yellow to blue or green. Figure 4 shows the reduction activity of the cell-free supernatant of strains. E. canintestini S18A showed the highest reducing power followed by E. dispar S27A, E. dispar S20B, $E$. canintestini SB3, and E. dispar S26A. Most of the strains showed desirable reducing properties with a significant difference $(p<0.05)$ as compared to the standard L. casei Shirota and Trolox.

The DPPH radical scavenging activity was explored for the cell-free supernatants. Figure 5 shows that strain $E$. canintestini S26B exhibited the highest scavenging activity of 57.98\%; others (30-50\%) while E. dispar S27A (6.146\%) and $E$. dispar S27B (3.85\%) exhibited the least activity. However, standard L. casei Shirota displayed $24.95 \%$ of radical scavenging property. The interaction of DPPH radicals with proton-donating antioxidants causes a decrease in the absorbance indicating scavenging property.

The cell-free supernatants were screened for their ability to scavenge the ABTS radical. The strain E. dispar S26A showed the highest inhibitory activity (88.36\%) against the radical (Fig. 6). All the tested strains and the standard strain displayed desirable inhibitory activity above $83 \%$. Many strains exhibited higher activity with significant difference than $L$. casei Shirota $(85.115 \%, p<$ $0.05)$ and were close to Trolox (87.64\%) reduction

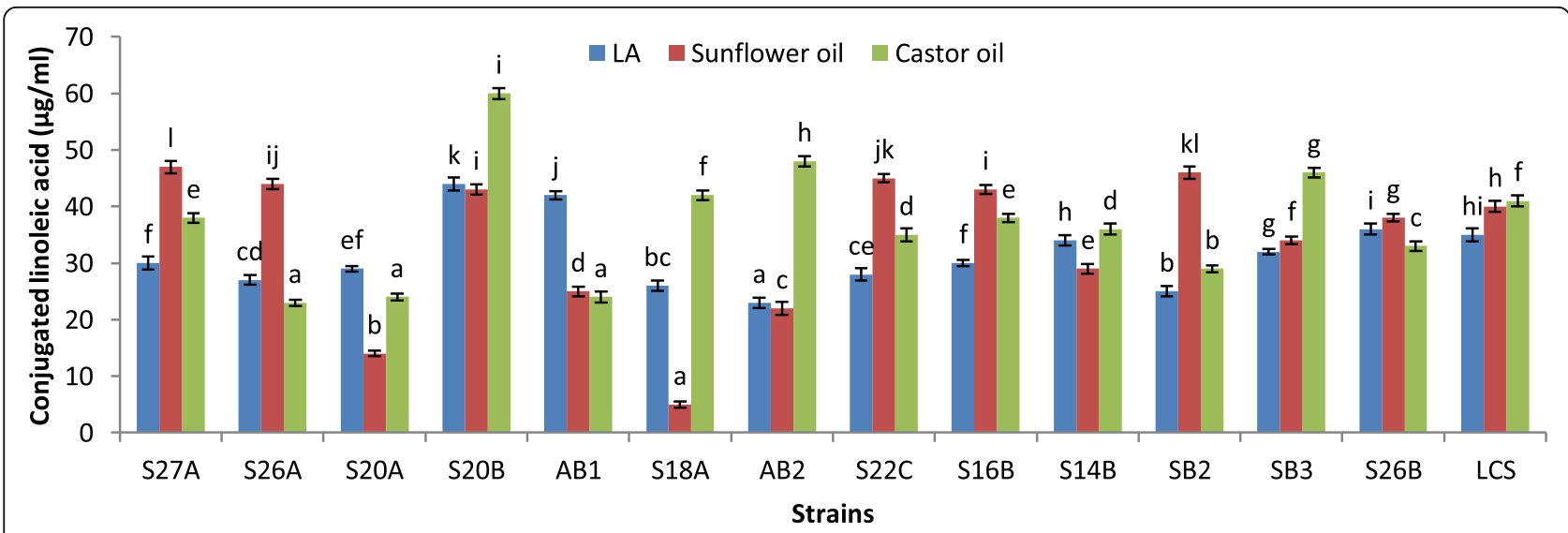

Fig. 2 Production of conjugated linoleic acid from linoleic acid-rich substrates like sunflower oil and castor oil. Highest yield was seen in castor oil by strain S20B. Castor and sunflower oil both can be used as natural substrates for production of CLA 


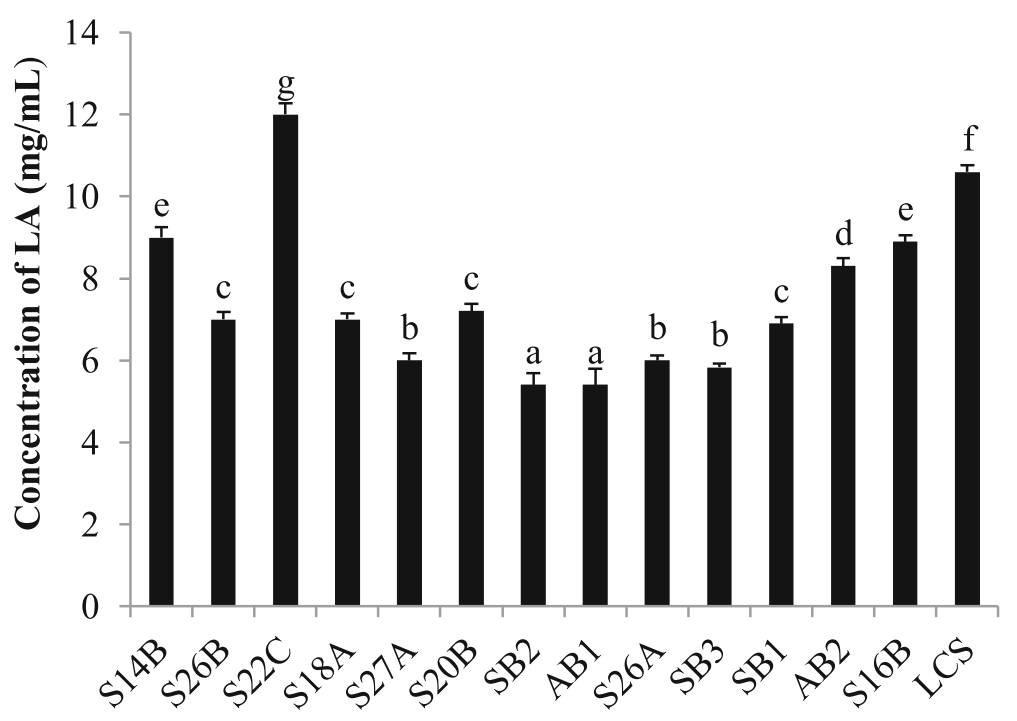

Strains

Fig. 3 Quantification of lactic acid produced by Enterococcus strains by titrimetric method. Strain S22C revealed highest production (12 mg/ml) of lactic acid

activity. In this assay, ferryl myoglobin radicals are formed which lead to the oxidation of ABTS to $\mathrm{ABTS}^{+}$radicals giving color. In the presence of an antioxidant, the reaction is suppressed to give a colorless solution [49].

\section{Discussion}

The enzymatic activities of organisms play an important role in the food processing industry. The fermentation processes involve the secretion of various useful enzymes by the organisms. Hence, the screening of the bacteria for the enzyme production is necessary for food processing. Though the organisms produce useful enzymes, they also tend to produce harmful enzymes. Several lactic acid bacteria (LAB) used in the food industry are well-known for producing the desired enzymes. Hence, the qualitative analysis is of prime importance in sorting out the best organisms. The qualitative analysis sometimes gives false-positive results. Thus, biochemical assays serve in quantifying and detecting minute amounts of enzyme [36].

The bile hydrolysis is an important feature of the lactic acid bacteria. The cholesterol in the human body is

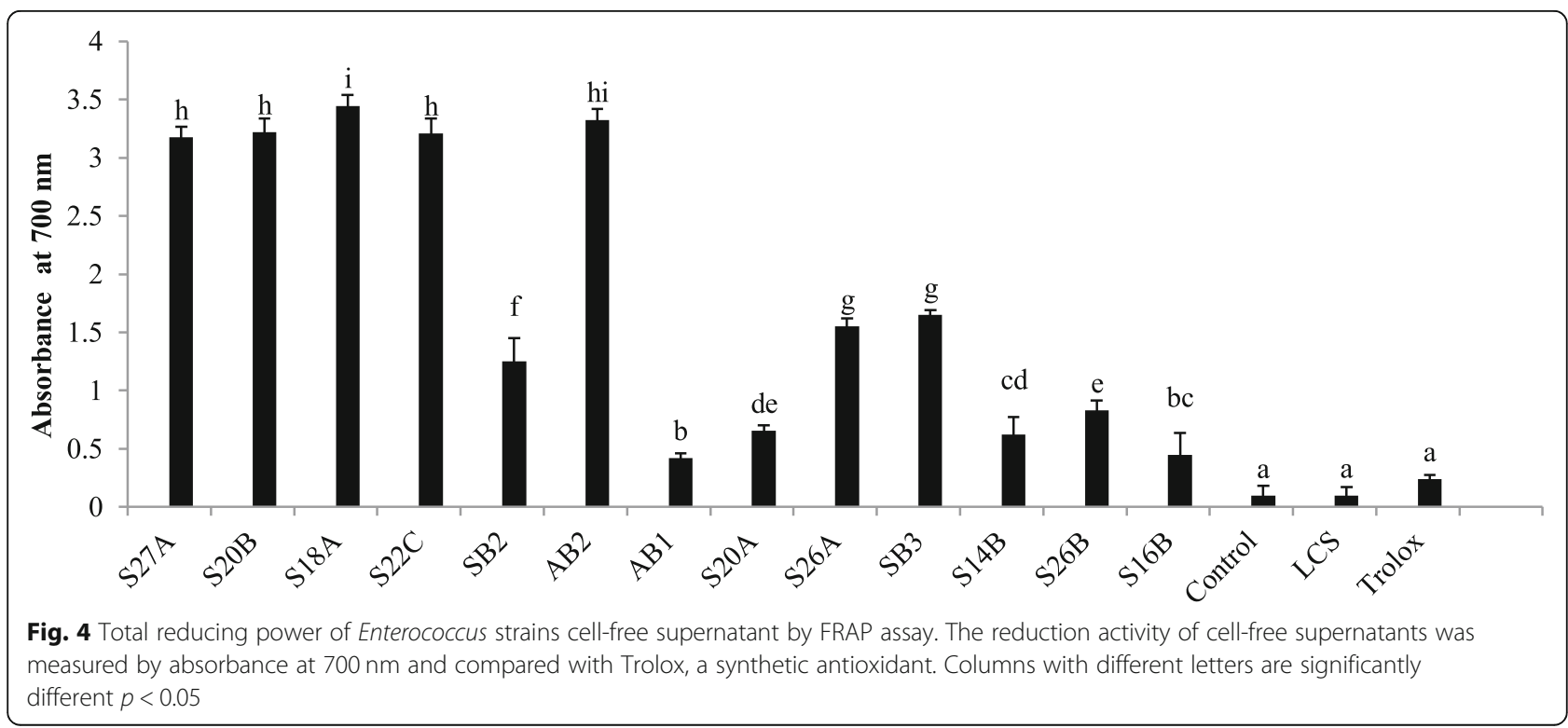




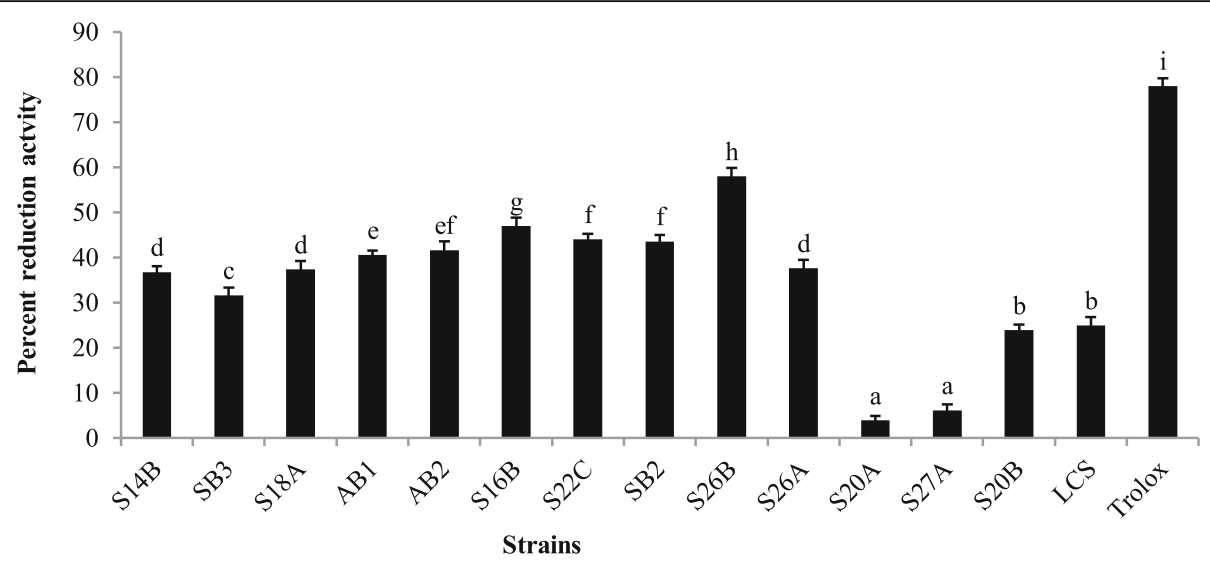

Fig. 5 Antioxidant activity of Enterococcus cell-free supernatant by DPPH free radical scavenging assay. Columns with different letters are significantly different $p<0.05$

stored in the form of conjugated bile salts and is excreted in the form of feces. Some fraction of these salts is not absorbed by the body and is released as free bile salts in the body. Thus, the deconjugation of bile salts can help in lowering the serum cholesterol levels in the lumen. The BSH enzyme produced by the bacteria helps maintain cholesterol levels in the body. Also, in order to survive the adverse conditions of the GIT, the bacteria produce bile salt hydrolase enzyme. These enzymes are produced as a defense mechanism by the bacteria against the bile salts [28]. Bile is secreted by the liver and attacks the bacteria by acting as detergents that dissolve the bacterial cell wall proteins. This is an intracellular enzyme and catalyzes bile salt hydrolysis. In a reported study, E. faecium EM 485 and EM 925 could hydrolyze bile salts in the medium [28]. Several lactic acid bacteria, as well as gut microbes, are reported to possess this enzyme activity [37]. The gut microbiota act on the intestinal bile by processes like deconjugation, dehydrogenation, deglucoronidation. Thus, the bile tolerance of the probiotic bacteria can be elucidated by the presence of this enzyme and it becomes a necessity factor of probiotic selection procedure [38].

Biogenic amines are generally found in poultry products, fish, meat, eggs, and fermented products like cheese and pickles. They are nitrogenous compounds produced by the body to confer physiological function of the immune response, brain, cell division, etc. But when these compounds when consumed orally, they prove to be toxic when taken in large quantities. Several researchers have reported the role of lactic acid bacteria in the production of biogenic amines. Thus, there is a possibility of production of these toxins during food processing. But, the production of these toxic amines takes place only after

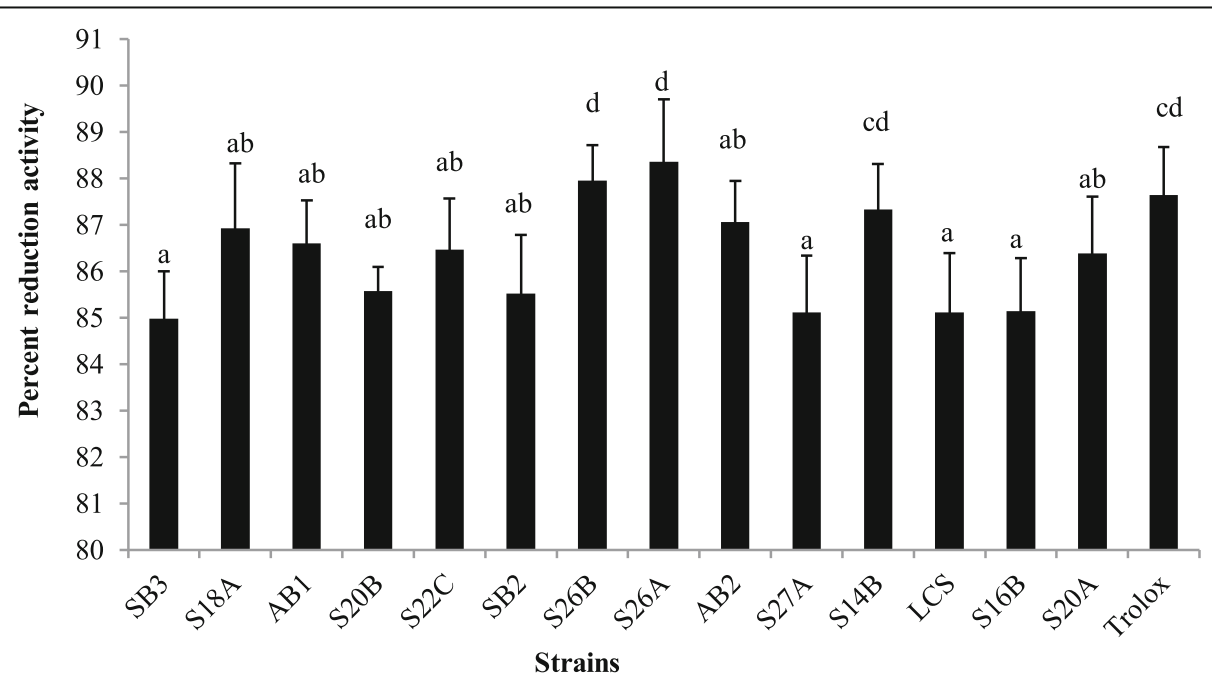

Fig. 6 Antioxidant activity of Enterococcus cell-free supernatant by ABTS free radical scavenging assay. Columns with different letters are significantly different $p<0.05$ 
the bacteria has sought favorable conditions during processing and storage. The bacteria produce the decarboxylases only when free amino acids or protein-rich foodstuff are available. Thus, the development and modification of these parameters and vigilant screening of good bacterial strains might help in eradicating the involvement of such toxic metabolites $[39,40]$.

Amylase enzyme is necessary for the degrading of starch molecules, thus helping in smooth digestion. Lactobacillus cultures were reported to produce this enzyme in large quantities in the gastrointestinal transit in chickens. This enzyme is produced both intracellularly as well as extracellularly [42]. The proteolytic activity of Enterococcus is present in the extracellular component of the bacteria. This activity is attributed to the metalloproteinase activity that hydrolyzes casein in the milk. Several studies have reported the presence of this enzyme in large quantities during ripening of cheese. The inoculum dose of the bacteria or the enzyme must be controlled in the process of dairy products as greater amounts of enzyme lead to flavor and aroma defects in the products [36]. Proteases and lipases help in biofilm formation of the probiotic organism, thus inhibiting the pathogens from the GIT [41]. In a recent study presenting synergistic effects of lipase and enterocin production, EF-5653 showed maximum activity of $6 \mathrm{U} \mathrm{ml}^{-1}$ and EF64 with $4 \mathrm{U} \mathrm{ml}^{-1}$ whereas standard E. faecium M5153 had no lipase activity [43]. All the strains reported in the present study showed excellent lipase activity when compared in the literature. This study reported the highest amounts of lipase activity by Enterococcus strains. The food industry involves the usage of lipases in the processing of cheese, dairy products, etc. The lipases secreted by these organisms also impart flavor and aroma to the product [42]. Certain studies have revealed the lethal effect of lipases on pathogens. The lipases acting synergistically with bacteriocins attack the lipid membrane of the bacterial cell, thus dissolving and helping in cell death [43].

Safflower oil, rice bran oil, palm oil, castor oil, sunflower oil, and soybean oil containing linoleic acid are natural sources of linoleic acid. Castor oil (90\% ricolenic acid) and sunflower oil (at least 69\%) are known for their rich linoleic acid content $[44,45]$. Hence, these oils were selected and examined for their biochemical conversion of linoleic acid by enterococci. This is the first study to report excellent CLA production by enterococci using natural substrates. Hosseini et al. have reported the use of these substrates for CLA conversion using L. plantarum [46]. The addition of Tween 80 to the substrate may aid in lipase production which in turn frees linoleic acid from the oils, thus hastening the process of biochemical conversion of CLA. Several studies using Lactobacillus, Bifidobacterium, and Propionibacterium reveal that these bacteria are known to possess linoleic acid isomerase which converts ricolenic and linolenic acid to CLA isomers [12].

Lactic acid is a frequently used chemical in the food industry, a flavoring agent, preservative, and acidulant. It also has application in the textile and pharmaceutical industry [47]. Lactic acid production by microorganisms is a mostly favored process than the chemical one as it prevents environmental pollution [48]. Fowoyo and Ogunbanwo carried out a similar study involving five Lactobacillus strains of which the highest quantity of lactic acid $\left(8 \mathrm{gl}^{-1}\right)$ was produced by $L$. lactis spp. [15].

All the strains showed higher reduction activity both by DPPH and ABTS assay when compared with the L. casei Shirota strain. In a similar study, E. durans LAB 18s strain exhibited high ABTS (9.4\%) and DPPH activity $\mathrm{EC}_{50}=3.6$, respectively [50]. Pieniz et al. also reported the antioxidant activity of E. faecium strains $\left(\mathrm{ABTS}=59-92.5 \% ; \mathrm{EC}_{50}=\right.$ $2.41-5.02 \mu \mathrm{g} / \mathrm{ml}$ ) isolated from meat and dairy products [51]. The consumption of radical scavenging probiotics has proven to be helpful in boosting the immunity of the host. The oxidative stress is caused by infectious agents and disorders which harm and deteriorate the cells. The probiotic bacteria are known to possess this activity. This attribute is contributed by the secondary antimicrobial compounds produced by the probiotic strain. The antioxidant activity of the probiotic bacteria helps in reducing the aging process, ulcers, cardiovascular disease, diabetes, and urinary tract infections. Recently, markets are flooded with synthetic carcinogenic antioxidants. Though these antioxidants help in slowing down the oxidation process, they may pose as a threat to the host. Consumption of bio-therapeutic microorganisms can scavenge the ROS radicals and save the body from its ill-effects. The cell-free supernatants of lactic acid bacteria have already proved their scavenging properties and thus have reduced the risk of chronic diseases [52]. The scavenging property of probiotics can be attributed to metal chelation, ROS radical scavenging, or ascorbate auto-oxidation activity [53]. The ROS theory proposes the development of antioxidative agents that would help in curbing the progression of free radical-related disorders.

\section{Conclusion}

The present investigation revealed enzymes and secondary metabolites of therapeutic as well as industrial value. The screening of metabolites produced by indigenous flora also helps in studying the intestinal metabolism and interaction of the microbiota. Excellent antioxidant activities of E. canintestini S26B and E. dispar S26A proved the scavenging potential of these strains. They can be used along with other well-known probiotic strains after clinical trials. The production various enzymes like protease and lipase promise value of these 
strains in dairy and food-beverage fermentation industries. Their property to produce conjugated linoleic acid using castor oil shows its future potential in production and use of these metabolites in nutrition studies. Further studies will involve optimization and production of important industrial enzymes and specific type of CLA produced by enterococci strains along with in vivo studies to confirm their probiotic potential.

\section{Abbreviations}

ABTS: 2,2-Azino-bis (3-ethylbenzothiazoline-6-sulphonic acid) diammonium salt; AOAC: Association of Analytical Communities; BA: Biogenic amines; $\mathrm{BHI}$ : Brain Heart Infusion broth; BSH: Bile salt hydrolase; CLA: Conjugated linoleic acid; DPPH: 1,1-Diphenyl-2-picrylhydrazyl; FAO: Food and Agriculture Organization; $\mathrm{FeCl}_{3}$ : Ferric chloride; FRAP: Ferric reducing antioxidant power; L. casei YIT 9029: Lactobacillus casei Shirota; LAB: Lactic acid bacteria; MRS: Mann-Rogosa-Sharpe; NaOH: Sodium hydroxide; ONPG: O-nitrophenol galactosidase; PBS: Phosphate-buffered saline; ROS: Reactive oxygen species; TCA: Trichloroacetic acid

\section{Acknowledgements}

We would like to thank Department of Obstetrics and Gynecology, Lokmanya Tilak Municipal Corporation General Hospital, Mumbai, India, for providing samples required for the project.

\section{Authors' contributions}

$A B$ performed the experiments and wrote the manuscript. USA supervised the work and edited the manuscript. All authors have read and approved the manuscript.

\section{Funding}

This work was funded by University Grants Commission-Basic Science Research (UGC-BSR), India. The funds were used to buy media and chemicals required for the project.

\section{Availability of data and materials \\ Not applicable.}

\section{Ethics approval and consent to participate}

The study was approved by Ethics Committee of Lokmanya Tilak General Municipal Corporation Hospital, Mumbai, India, with reference no: IEC/04/13. A written and informed consent was obtained from all the subjects.

\section{Consent for publication}

N/A

\section{Competing interests}

All authors declare that they have no competing interests.

Received: 8 August 2019 Accepted: 23 September 2019 Published online: 25 November 2019

\section{References}

1. Bhagwat A, Nandanwar YA, Warke R, Annapure US (2019) In vitro assessment of physiological properties of Enterococcus strains of human origin for possible probiotic use. Asian J pharm Clin Res 12

2. Tallon R, Arias S, Bressollier P, Urdaci MC (2007) Strain- and matrixdependent adhesion of Lactobacillus plantarum is mediated by proteinaceous bacterial compounds. J Appl Microbiol. https://doi.org/10 1111/j.1365-2672.2006.03086.x

3. Bouzaine T, Dauphin RD, Thonart P, Urdaci MC, Hamdi M (2005) Adherence and colonization properties of Lactobacillus rhamnosus TB1, a broiler chicken isolate. Lett Appl Microbiol. https://doi.org/10.1111/j.1472-765X. 2005.01684.x

4. Arias LG, Fernández D, Sacristán N, Arenas R, Fresno JM, Tornadijo E (2013) Enzymatic activity, surface hydrophobicity and biogenic amines production in lactic acid bacteria isolated from an artisanal Spanish cheese. 7:21142118. https://doi.org/10.5897/AJMR2012.2288
5. Hayek SA, Shahbazi A, Worku M, Ibrahim SA (2013) Enzymatic activity of Lactobacillus reuteri grown in a sweet potato based medium with the addition of metal ions. Springerplus:1-10

6. Kučerová K, Svobodová H, Tůma Š, Ondráčková I (2009) Production of biogenic amines by enterococci. 27:50-55

7. Irastorza A (2007) Biogenic amine production by lactic acid bacteria isolated from cider. 45:473-478. https://doi.org/10.1111/j.1472-765X.2007.02207.x

8. Bassaganya-Riera J, Hontecillas R, Beitz DC (2002) Colonic anti-inflammatory mechanisms of conjugated linoleic acid. Clin Nutr. https://doi.org/10.1054/ clnu.2002.0594

9. Belury M (2002) Inhibition of carcinogenesis by conjugated linoleic acid: potential mechanisms of action. J Nutr

10. Gaullier JM, Halse J, Hoye K, Kristiansen K, Fagertun H (2004) Conjugated linoleic acid supplementation for 1 y reduces body fat mass in healthy overweight humans. Am J Clin Nutr

11. Thom E, Wadstein J, Gudmundsen O (2001) Conjugated linoleic acid reduces body fat in healthy exercising humans. J Int Med Res. https://doi. org/10.1177/147323000102900503

12. Sieber R, Collomb M, Aeschlimann A, Jelen P, Eyer H (2004) Impact of microbial cultures on conjugated linoleic acid in dairy products - a review. 14:1-15. https://doi.org/10.1016/S0958-6946(03)00151-1

13. Ushe TC, Nagy B (1985) Inhibition of small intestinal colonization of enterotoxigenic Escherichia coli by streptococcus faecium M74 in pigs. Zentralbl Bakteriol Mikrobiol Hyg [B]

14. Chisari G, lo Bue AM, Drago L, Abbiati R, Castiglioni G, Gismondo MR. Role of Enterococcus faecium SF 68 in experimental infection in mice. G Ital Chemioter 1989

15. Fowoyo PT, Ogunbanwo ST (2010) Phenotypic diversity of lactic acid bacteria isolated from Massa, a fermented maize dough. 4:2682-2691

16. Samad MY a., Razak CN a., Salleh AB, Zin Wan Yunus WM, Ampon K, Basri M. A plate assay for primary screening of lipase activity. J Microbiol Methods 1989. doi:https://doi.org/10.1016/0167-7012(89)90030-4

17. Harley JP\& LMP. Laboratory Exercises in Microbiology, Fifth Edition. McGraw -Hill Companies, Inc, New York 2002. doi:https://doi.org/10.1007/s13398014-0173-7.2

18. Lloyd AB, Sheaffe J (1973) Urease activity in soils. Plant Soil. https://doi.org/ 10.1007/BF00018046

19. Cheesbrough M. Oxidase Test. Dist. Lab. Pract. Trop. Countries, Part 2, 2006

20. American Society for Microbiology. Oxidase Test Protocol. ASM MicribeLibrary 2013

21. Mugg P (1983) A rapid hippurate hydrolysis test for the presumptive identification of group B streptococci. Pathology. https://doi.org/10.3109/ 00313028309083502

22. Qadri SMH, DeSilva MI, Zubairi S (1980) Rapid test for determination of esculin hydrolysis. J Clin Microbiol. https://doi.org/10.1007/BF00394656

23. Kaushik JK, Kumar A, Duary RK, Mohanty AK, Grover S, Virender K (2009) Functional and probiotic attributes of an indigenous isolate of Lactobacillus plantarum. 4. https://doi.org/10.1371/journal.pone.0008099

24. Mete A, Coşansu S, Demirkol O, Ayhan K (2017) Amino acid decarboxylase activities and biogenic amine formation abilities of lactic acid bacteria isolated from shalgam. Int J Food Prop. https://doi.org/10.1080/10942912. 2016.1152479

25. Nguyen T, Splechtna B, Krasteva S (2007) Characterization and molecular cloning of a heterodimeric $b$-galactosidase from the probiotic strain Lactobacillus acidophilus R22. 269:136-144. https://doi.org/10.1111/j.15746968.2006.00614.x

26. Sakthi SS, Kanchana D, Saranraj P, Usharani G (2012) Evaluation of amylase activity of the amylolytic fungi Aspergillus niger using cassava as substrate. Int J Appl Microbiol Sci

27. Takahashi J, Imada Y, Yamada K (1963) Lipase formation by micro-organisms grown on hydrocarbons. Nature 200:1208

28. Maria K, Diogo A, Vieira S (2015) Safety, beneficial and technological properties of Enterococcus faecium isolated from Brazilian cheeses. 249:237-249

29. Barrett E, Ross RP, Fitzgerald GF, Stanton C (2007) Rapid screening method for analyzing the conjugated linoleic acid production capabilities of bacterial cultures. 73:2333-2337. https://doi.org/10.1128/AEM.01855-06

30. (AOAC), International A of OAC. Official methods of analysis of the Association of Official Analytical Chemists International. 2012

31. Wu D, Sun MZ, Zhang C, Xin Y (2014) Antioxidant properties of Lactobacillus and its protecting effects to oxidative stress caco-2 cells. 24 : $1766-1771$ 
32. Liu H, Lin Y (2013) Applications of Lactobacillus rhamnosus spent culture supernatant in cosmetic antioxidation, whitening and moisture retention applications:14161-14171. https://doi.org/10.3390/molecules181114161

33. Re R, Pellegrini N, Proteggente A, Pannala A, Yang M, Rice-Evans C (1999) Antioxidant activity applying an improved ABTS radical cation decolorization assay. Free Radic Biol Med. https:/doi.org/10.1016/S0891-5849(98)00315-3

34. Sonawane S, Arya SS (2013) Antioxidant activity of jambhul, wood apple, ambadi and ambat chukka: an indigenous lesser known fruits and vegetables of India. Adv J Food Sci Technol

35. Manero A, Blanch AR (1999) Identification of Enterococcus spp. with a biochemical key. 65:4425-4430

36. Arizcun C, Barcina Y, Torre P (1997) Identification and characterization of proteolytic activity of Enterococcus spp . isolated from milk and Roncal and Idiazabal cheese. 38:17-24

37. Glibowski P, Targon Z (2014) A new insight into the physiological role of bile salt hydrolase among intestinal bacteria from the genus Bifidobacterium:1-24. https://doi.org/10.1371/journal.pone.0114379

38. Hill C, Gahan CGM (2006) Bile salt hydrolase activity in probiotics. 72:1729-1738. https://doi.org/10.1128/AEM.72.3.1729

39. Gardini F, Martuscelli M, Caruso MC (2001) Effects of pH, temperature and $\mathrm{NaCl}$ concentration on the growth kinetics, proteolytic activity and biogenic amine production of Enterococcus faecalis. 64:105-117

40. Kalhotka L, Manga I, Přichystalová J, Hůlová M, Vyletělová M, Šustová K. Decarboxylase activity test of the genus Enterococcus isolated from goat milk and cheese The ability to decarboxylate amino acids is characteristic for many microorganisms, especially for genera of lactic acid bacteria (M aijala et al 1993 ; De L lano 2012:145-51. doi:https://doi.org/10.2754/avb201281020145

41. Hussain M, Khan M (2015) Technological characterization of indigenous Enterococcal population for probiotic potential

42. Jin LZ, Ho YW, Abdullah N, Jalaludin S. Digestive and Bacterial Enzyme Activities in Broilers Fed Diets Supplemented with Lactobacillus Cultures 1987:886-891

43. Ramakrishnan V, Narayan B, Halami PM (2012) Combined Effect of Enterocin and Lipase From Enterococcus faecium NCIM5363 against food borne pathogens: mode of action studies:162-169. https://doi.org/10.1007/s00284-012-0138-z

44. Colakoglu H, Gursoy O (2011) Effect of lactic adjunct cultures on conjugated linoleic acid (CLA) concentration of yogurt drink. J Food Agric Environ.

45. Chin SF, Liu W, Storkson JM, Ha YL, Pariza MW (1992) Dietary sources of conjugated dienoic isomers of linoleic acid, a newly recognized class of anticarcinogens. J Food Compos Anal. https://doi.org/10.1016/08891575(92)90037-K

46. Hosseini ES, Kermanshahi RK (2015) Conjugated linoleic acid production from various substrates by probiotic Lactobacillus plantarum:27-32. https:// doi.org/10.1007/s13213-014-0832-0

47. Varadarajan S, Miller DJ (1999) Catalytic upgrading of fermentation-derived organic acids. Biotechnol Prog. https://doi.org/10.1021/bp9900965

48. Åkerberg C (2000) An economic evaluation of the fermentative production of lactic acid from wheat flour. Bioresour Technol. https://doi.org/10.1016/ S0960-8524(00)00057-2

49. Curiel JA, Pinto D, Marzani B (2015) Lactic acid fermentation as a tool to enhance the antioxidant properties of Myrtus communis berries. Microb Cell Factories 14:67. https://doi.org/10.1186/s12934-015-0250-4

50. Pieniz S, Andreazza R, Anghinoni T, Camargo F, Brandelli A (2014) Probiotic potential, antimicrobial and antioxidant activities of Enterococcus durans strain LAB18s. Food Control 37:251-256. https://doi.org/10.1016/j.foodcont.2013.09.055

51. Pieniz S, Andreazza R, Okeke BC, Camargo FAO, Brandelli A (2015) Antimicrobial and antioxidant activities of Enterococcus species isolated from meat and dairy products. Braz J Biol. https://doi.org/10.1590/1519-6984.02814

52. Xing J, Wang G, Zhang Q, Liu X, Gu Z, Zhang H (2015) Determining antioxidant activities of lactobacilli cell-free supernatants by cellular antioxidant assay: a comparison with traditional methods:1-16. https://doi. org/10.1371/journal.pone.0119058

53. Nazari F, Delborde Y, Karimitabar Z, Ranjbar A, Alikhani MY (2016) Antioxidant effect of Lactobacillus acidophilus as a probiotic at different time intervals. Avicenna J Clin Microbiol Infect 3:10-12. https://doi.org/10. 17795/ajcmi-34575

\section{Publisher's Note}

Springer Nature remains neutral with regard to jurisdictional claims in published maps and institutional affiliations.

\section{Submit your manuscript to a SpringerOpen ${ }^{\circ}$ journal and benefit from:}

- Convenient online submission

- Rigorous peer review

- Open access: articles freely available online

- High visibility within the field

- Retaining the copyright to your article

Submit your next manuscript at $\boldsymbol{\nabla}$ springeropen.com 